\title{
Multi Program-Components Handshaking (MPH) Utility Version 3 User's Manual
}

\author{
Yun He And Chris Ding \\ NERSC Division, Lawrence Berkeley National Laboratory
}

June 20, 2002

\section{Contents}

1 Introduction $\quad 3$

2 How to Use 5

3 How to Compile and Run 5

$\begin{array}{lll}4 & \text { Acknowledgement } & 7\end{array}$

5 Routine/Function Prologues $\quad \mathbf{8}$

5.1 Module MPH_module - Multi Program-Components Handshaking (Source

File: mph.F) ..................... 8

5.1.1 MPH_components - main MPH setup function . . . . . . . . . 10

5.1.2 MPH_init - initialize MPI and read the processors map info . . . . . 11

5.1.3 MPH_local - local handshaking . . . . . . . . . . . . . . . . . 12

5.1.4 MPH_global - global handshaking . . . . . . . . . . . . . 12

5.1.5 PE_in_component - check if a processor is in a component . . . . . . 13

5.1.6 PE_in_num_comps - return the number of components a processor . 14

5.1 .7 MPH_global_id - find global processor id . . . . . . . . . . . . . 15

5.1 .8 MPH_comm_join - join two components . . . . . . . . . . 15

5.1.9 MPH_redirect_output - redirect output from each component . . . . 16

5.1.10 MPH_read_list - read and process info from "processors_map.in" . . 17

5.1 .11 MPH_find_name - find name in a namelist . . . . . . . . . . . . 19

5.1 .12 MPH_help - display help info . . . . . . . . . . . . . . . 19

5.1.13 MPH_debug - define debug level . . . . . . . . . . . . . 20

5.1.14 MPH_timer - collect timing info in different channels. . . . . . . . 20

5.1.15 MPH_total_components - find number of total components . . . . . 21

5.1.16 MPH_comp_name - find component name given component id . . . 22

5.1.17 MPH_comp_id - find component id given component name . . . . . 23

5.1.18 MPH_local_world - find local communicator given component name 24

5.1.19 MPH_exe_id - find executable id given component name . . . . . . . 24

5.1 .20 MPH_total_num_exe - find total number of executables . . . . . . 25 
5.1.21 MPH_num_comps - find number of components in an executable . . 26

5.1.22 MPH_local_proc_id - find local processor id in a component . . . . . 26

5.1 .23 MPH_local_totProcs - find total number of processors . . . . . . . 27

5.1 .24 MPH_global_proc_id - find global processor id . . . . . . . . . . 28

5.1.25 MPH_global_proc_id - find total number of processors . . . . . . . 29

5.1.26 MPH_local_world - find local communicator of an executable . . . . 29

5.1.27 MPH_exe_low_proc_limit - find lower processor limit of a component 30

5.1.28 MPH_exe_up_proc_limit - find upper processor limit of a component 31 


\section{Introduction}

MPH version 2 combines all features of MPH version 1, unifies the interfaces, and provides more flexible components integration/execution modes.

In a distributed multi-component environment, each executable resides on a set of SMP nodes. Components within an executable may overlap on different nodes or processors.

MPH Version 2 contains the following functionality:

- component name registration

- resource allocation

- multi-component single executable, multi-component multi-executable, etc.

o inter-component communication

o inquiry on the multi-component environment

o standard in/out redirect

Please see more information at

http://www.nersc.gov/research/SCG/acpi/MPH

and please list the following in your reference if useful:

"MPH: a Library for Distributed Multi-Component Environment" Chris Ding and Yun He, Lawrence Berkeley Nat'l Lab Tech Report 47930, May 2001.

Consider the entire simulation system (CCSM) consists of many executables, each executable containing one or more components. This architecture offers complete flexibility, and is consistent with CORBA, DCE, CCA et al.

1) Every executable starts with

$$
\begin{aligned}
& \text { mpi_exec_world }= \\
& \text { MPH_components (name1='ocean', name2=' atmosphere',...) }
\end{aligned}
$$

You may have only one component in this executable, or up to 10 components in this executable. Component names are nametags (place holder) and are completely arbitrary. They must be self-consistently used, and match the "processors_map.in" registration file. This setup subroutine replaces MPH_setup() in MPH version 1. All other MPH functionality remains identical.

2) Some usages: 
a) CCSM example. Ice \& Land share one executable.

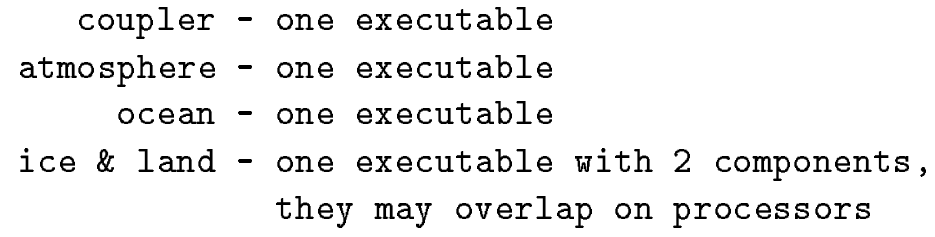

b) CCSM example. Multiple instances of atmosphere.

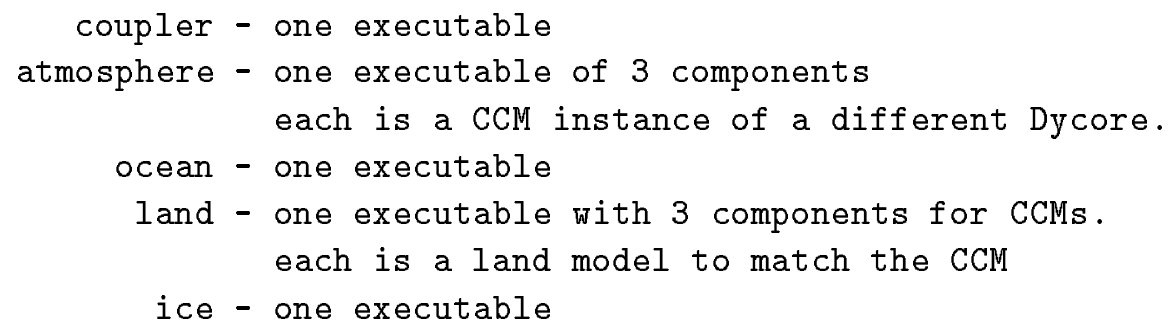


END

a) Allocation of processors for each executable is controlled by job launching process (different on IBM, SGI, Compaq).

b) Processor ranges for each components are defined local to the exeutable.

\section{How to Use}

Users need to "use MPH_module" in the application codes, and invoke the appropriate $\mathrm{MPH}_{-}$components function for the multiple components in each executable. For example, ICE_LAND_World = MPH_components (name1="ice", name2="land"). You could use MPH_debug call to determine the output message amount, the default level is 0. "MPH_help" call provides you the available inquiry functions for that mode.

An input file called "processors_map.in" to give detailed information of component nametags and processor ranges. See more detail about how this file looks like in Section 1.

Each component maintains its own output in a separate file (file name defined by environment variable either in command line or in batch run script), assuming the local processor 0 of each component being responsible for most output, other occasional writes from all the components are stored in one combined standand output file.

This is accomplished by processor rank 0 of each component call subroutine "MPH_redirect_output" with the model name as argument. IBM and SGI could do the output redirect with the help of system function "getenv" or "pxfgetenv". Compaq cannot do this. And T3E is able to get the correct output files created using "pxfgetenv", but only output with those "write $(6, *)$ " could be redirected, but not those with "write $(*, *)$ ", since $*$ is equal to unit 101, and permanently related to the non-redirectable stdout.

\section{How to Compile and Run}

The shared "Makefile" detects the machine architecture and 


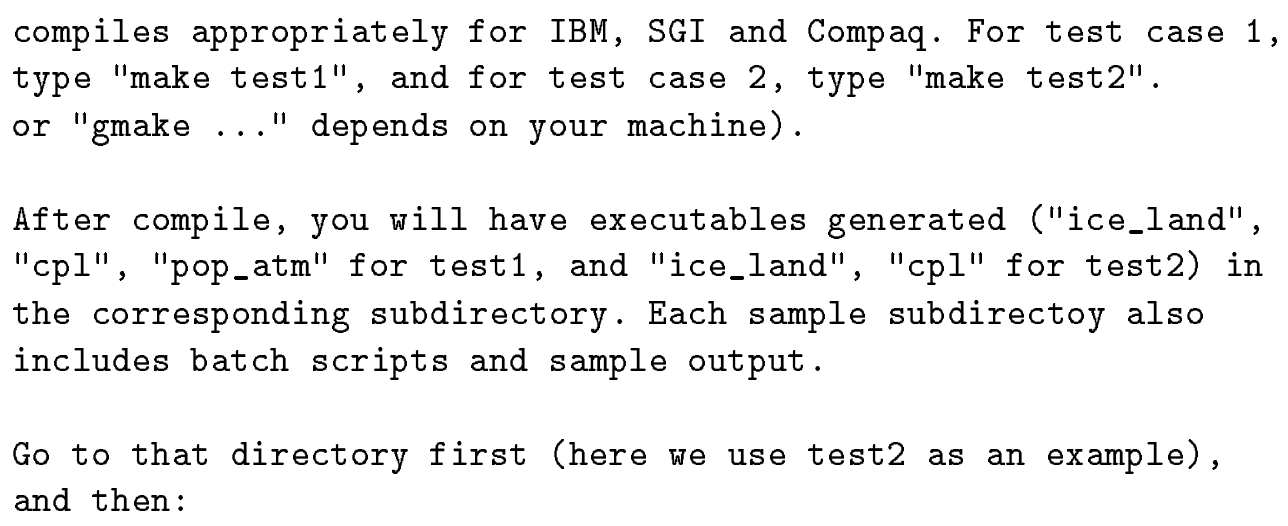

1) To run on NERSC and NCAR IBM SP interactively:

a) \% unsetenv MP_TASKS_PER_NODE

b) $\%$ setenv ice_out_env ice.log

$\%$ setenv land_out_env land.log

$\%$ setenv cpl_out_env cpl.log

c) Make sure the following command in ONE LINE:

$\%$ poe -pgmmodel mpmd -cmdfimle tasklist -nodes 3 -procs 6

-stdoutmode ordered -infolevel $2>\&$ output \&

This is to run the executables listed in user supplied "tasklist"

in the mpmd mode on total of 3 nodes and 6 procs.

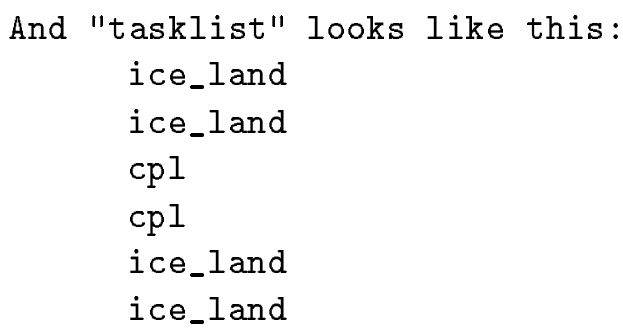

To run on IBM SP with batch script:

$\%$ 1lsubmit runscript.ibm

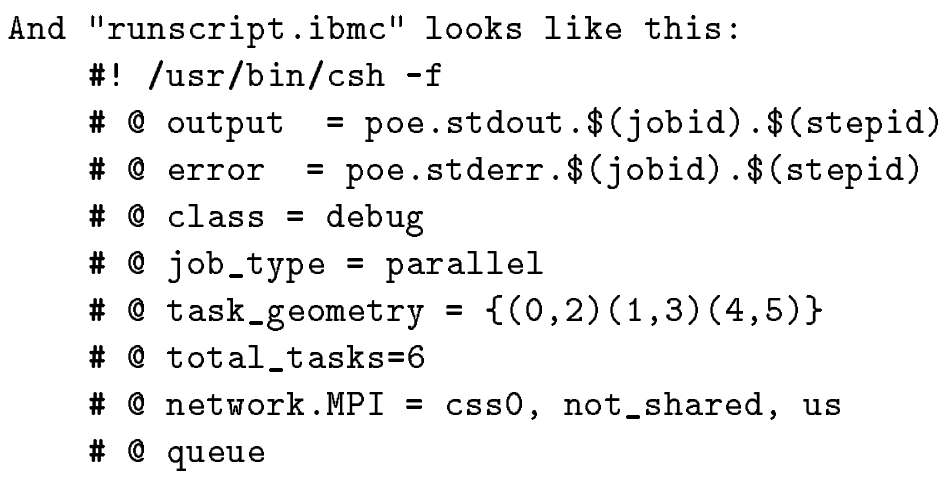




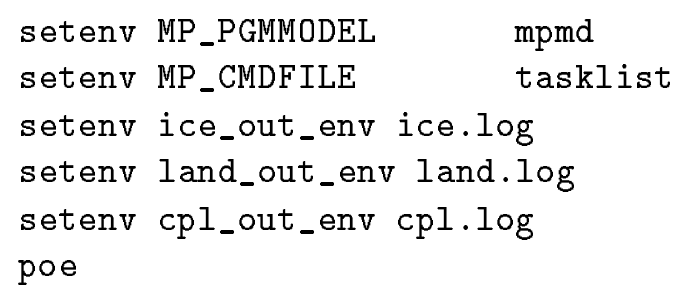

2) We could not run it on NERSC CRAY T3E since there is no mpmd mechnism .

3) To run on NCAR SGI interactively:

a) $\%$ setenv ice_out_env ice.log

$\%$ setenv land_out_env land.log

$\%$ setenv cpl_out_env cpl.log

b) \% mpirun $-\mathrm{p}$ "[\%g]" -np 4 ice_land : -np $2 \mathrm{cpl}>$ output

This is to run ice_land on 4 procs and cpl on 2 procs.

$[\% \mathrm{~g}]$ is to print the global id as a prefix for each output line.

4) To run on NCAR Compaq with batch script:

$\%$ prun -n6 -t runscript.dec

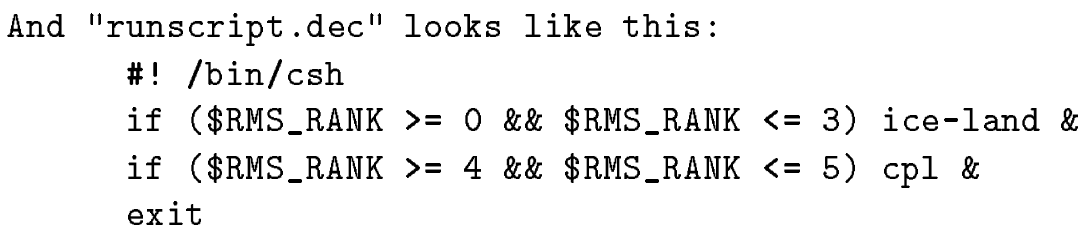

\section{Acknowledgement}

MPH is developed in collaboration with Tony Craig, Brian Kauffman, Vince Wayland and Tom Bettge of National Center of Atmospheric Research, and Rob Jacobs and Jay Larson of Argonne National Laborotory. Vince Wayland of NCAR contributes for Makefiles on SGI and Compaq. Dan Anderson and Bill Celmaster of NCAR also help in the batch run script of Compaq. This work is supported by the Office of Biological and Environmental Research, Climate Change Prediction Program, under ACPI Avant Garde project, and by the Office of Computational and Technology Research, Division of Mathematical, Information, and Computational Sciences, both of the U.S. Department of Energy under contract number DE-AC03-76SF00098. 


\section{Routine/Function Prologues}

\subsection{Module MPH_module - Multi Program-Components Handshaking (Source File: mph.F)}

This module multiple executables with multiple components in each executable. This module multiple executables with multiple components in.

\section{REVISION HISTORY:}

2001-Nov-15 -- add PROTEX convention

2001-May-20 -- first prototype

INTERFACE:

module MPH_module

USES:

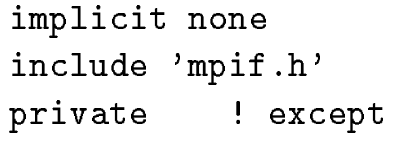

\section{PUBLIC MEMBER FUNCTIONS:}

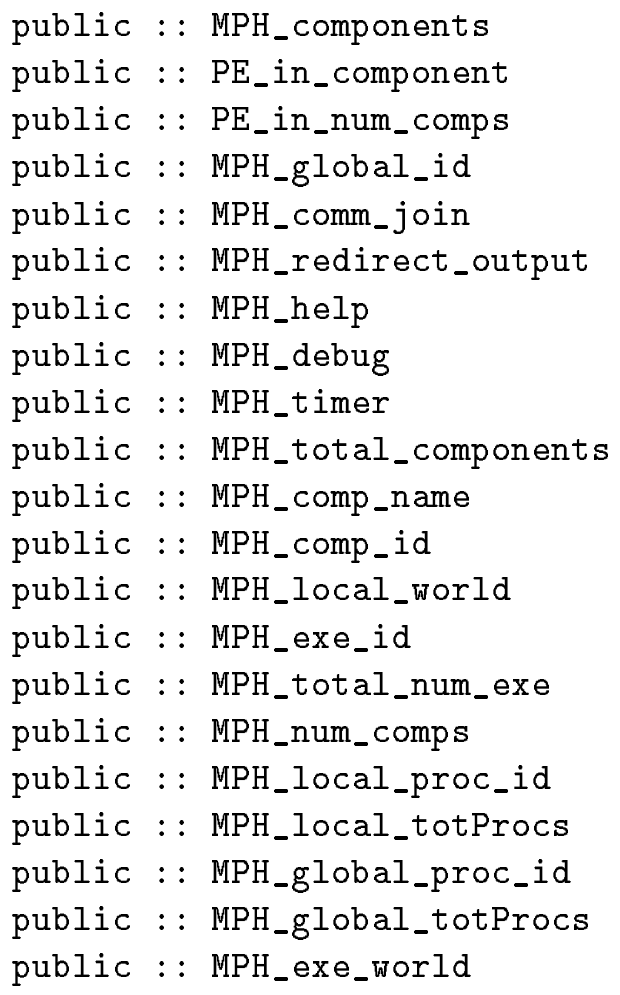


public : : MPH_exe_low_proc_limit

public : : MPH_exe_up_proc_limit

PUBLIC DATA MEMBERS:

integer, public : : istatus(MPI_STATUS_SIZE), ierr

integer, public : : MPH_Global_World ! total processor for the whole world

DEFINED PARAMETERS:

integer, parameter : : max_num_comps=10 ! maximum number of components

integer, parameter : : maxProcs_comp=128 ! maximum number of procs per comp

integer, parameter : : max_num_exes $=10$ ! maximum number of executables

integer, parameter : : N_CHANNELS $=10$ ! number of channels for timing

\section{LOCAL VARIABLES:}

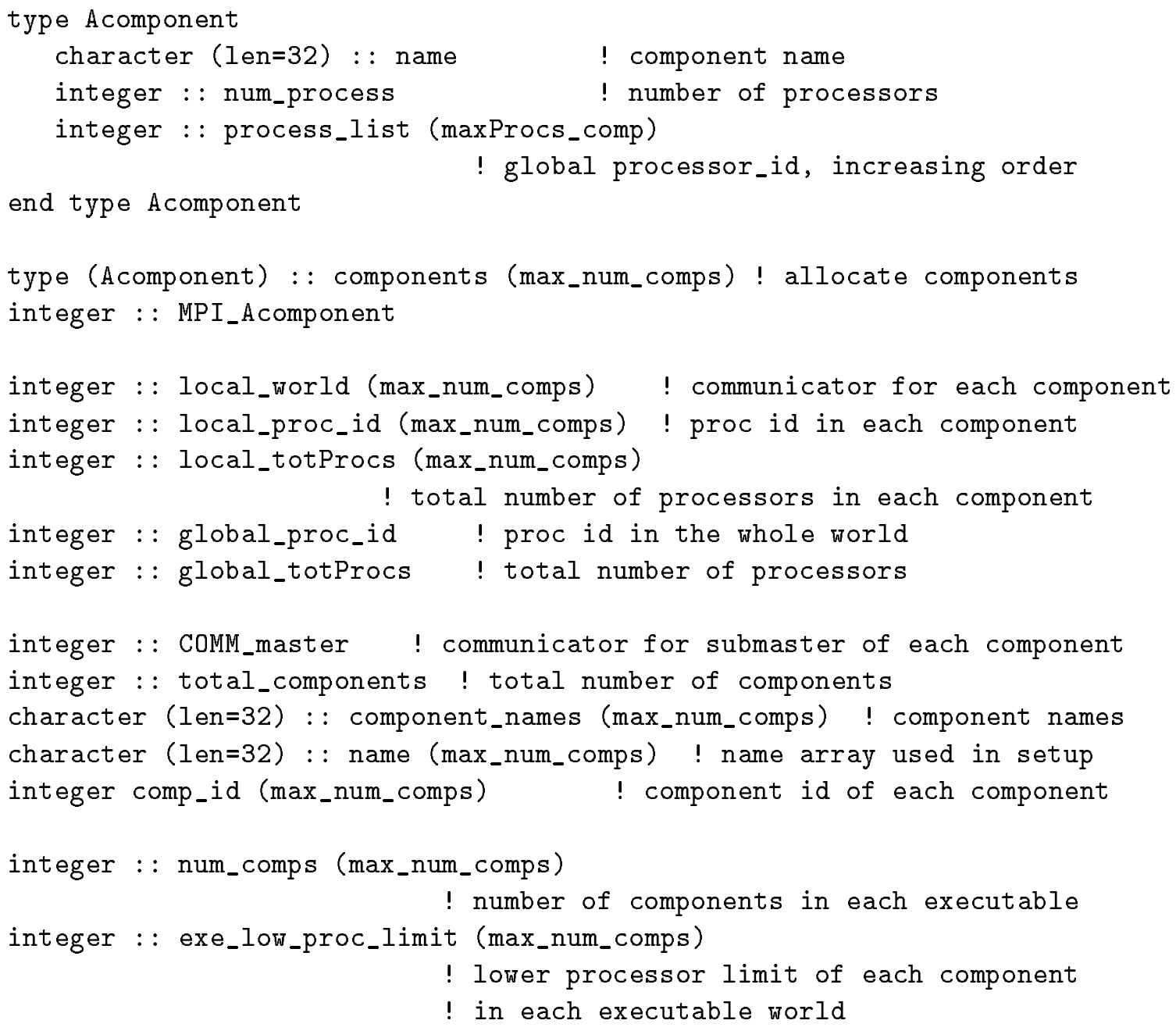




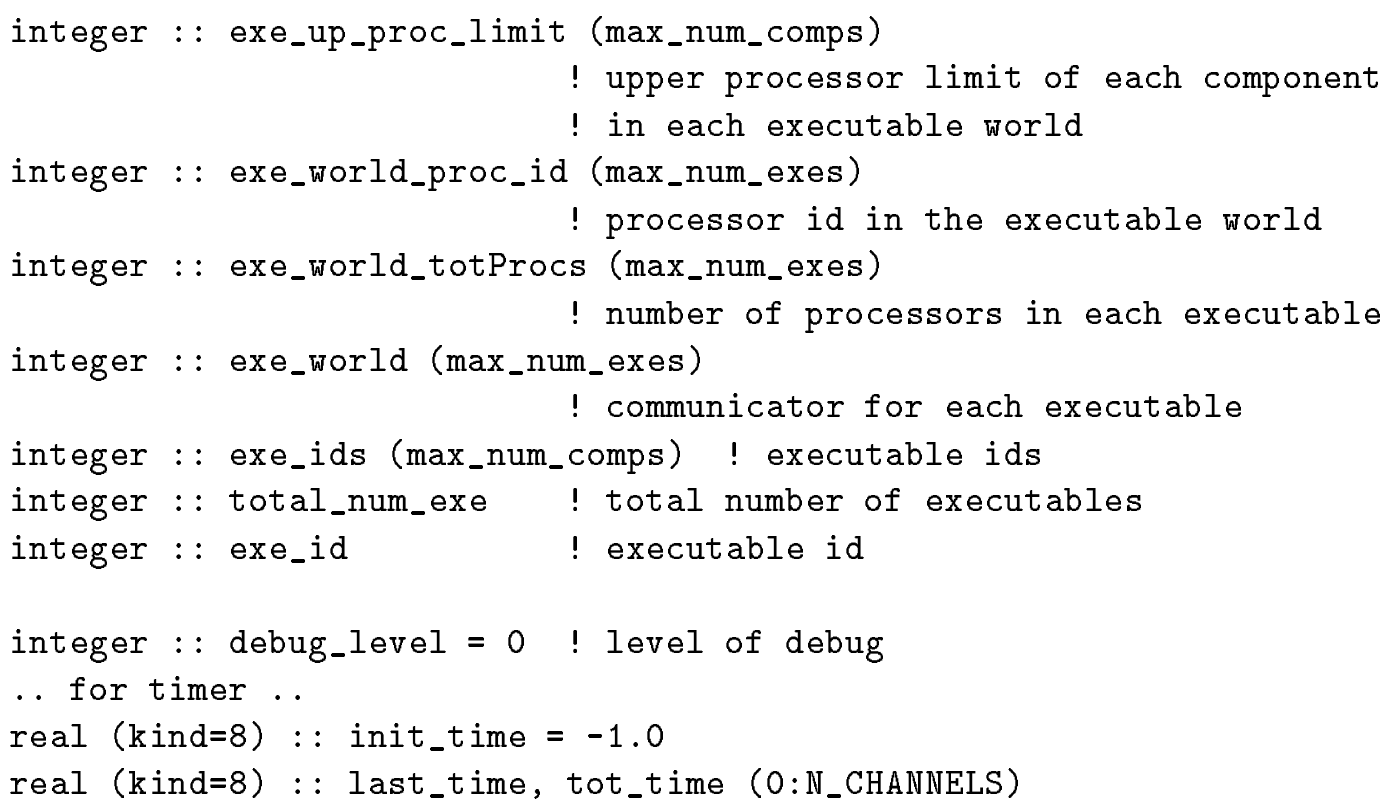

\subsubsection{MPH_components - main MPH setup function}

This is the main function for each of the executable to call to setup the distributed multicomponent environment. For example, if ocean and atmosphere sits in one executable, the source code will contain:

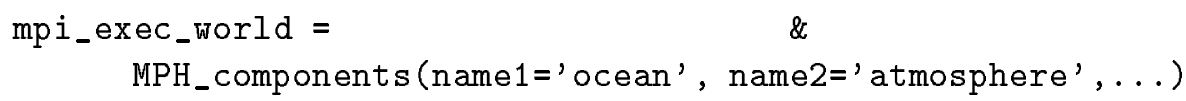

This function returns the MPI communicator of local executable world.

\section{REVISION HISTORY:}

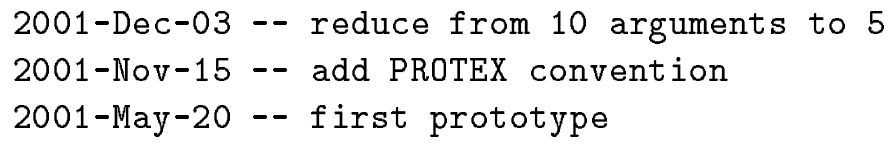

INTERFACE:

integer function MPH_components (name1, name2, name3, name4, name5)

USES:

implicit none 
INPUT PARAMETERS:

These are component names character (len=*), intent (in) : : name1

character(len=*), intent(in), optional : : name2, name3, name4, name5

OUTPUT PARAMETERS:

! This function returns the MPI communicator of this executable

! world: exe_world (exe_id)

SEE ALSO:

MPH_init, MPH_local, MPH_global, MPH_debug

LOCAL VARIABLES:

integer : : $\mathrm{k}$

\subsubsection{MPH_init - initialize MPI and read the processors map info}

This routine calls mpi_init, obtains global processor id. It reads and processes the "processors_map.in" file. It also defines an MPI_Acomponent sturcture (includes component name, number of processors and processor list for each component) for easy gather and scatter.

REVISION HISTORY:

2001-Nov-15 -- add PROTEX convention

2001-May-20 -- first prototype

INTERFACE:

subroutine $\mathrm{MPH}_{-}$init ()

USES:

implicit none

SEE ALSO:

MPH_read_list, MPH_local, MPH_global

LOCAL VARIABLES:

integer :: iblock(3), idisp(3), itype(3) 


\subsubsection{MPH_local - local handshaking}

This routine first defines exe_id, and creates local exe_world for each executable. It then gathers gobal processor ids onto submaster (whose rank is 0 in exe_world). And then it creates local_world for each component within exe_world based on its upper and lower processor limits. Finally it collects name, number of processors, and processor list of each component onto submaster of each executable world.

\section{REVISION HISTORY:}

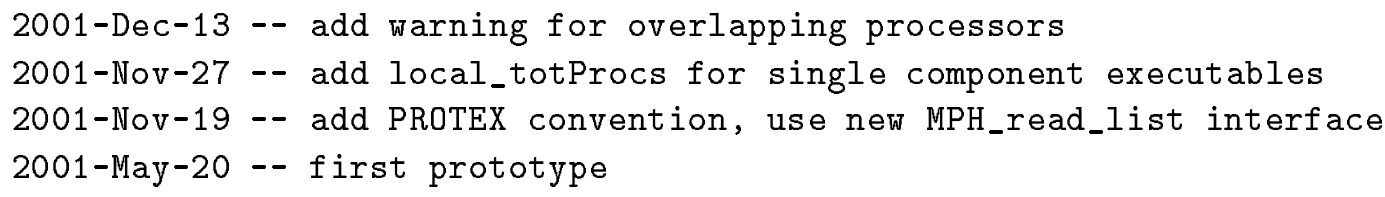

INTERFACE:

subroutine MPH_local ()

USES:

implicit none

SEE ALSO:

MPH_init, MPH_global, MPH_find_name

LOCAL VARIABLES:

integer : : color, key

integer : : id, comp_id_end, i, $k$

\subsubsection{MPH_global - global handshaking}

This routine first creates an MPI communicator COMM_master for all submasters (whose rank is 0 in the executable world). It then does an MPI_allgatherv in COMM_master to collect all the components information from each submaster. Then each submaster broadcasts AComponents to all PEs in its local exe_world. Finally, every processor lists the complete info of all the components.

\section{REVISION HISTORY:}




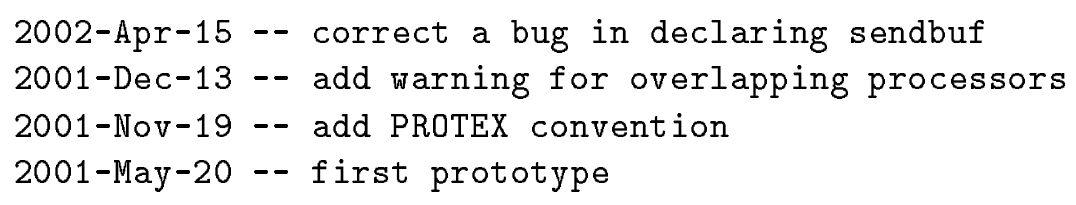

INTERFACE:

subroutine MPH_global ()

USES:

implicit none

SEE ALSO:

MPH_init, MPH_local, MPH_debug

\section{LOCAL VARIABLES:}

integer : : id, i, color, key

type (Acomponent) : : sendbuf (max_num_comps)

integer : : sendcount

integer :: recvcounts (0:total_num_exe-1)

integer : : displs (0:total_num_exe-1)

\subsubsection{PE_in_component - check if a processor is in a component}

This is a logical function to check if a processor is in a component.

REVISION HISTORY:

2001-Nov-15 -- add PROTEX convention

2001-May-20 -- first prototype

INTERFACE:

logical function $\mathrm{PE}_{-}$in_component (name, comm)

USES:

implicit none 
SEE ALSO:

MPH_find_name

INPUT PARAMETERS:

character(len=*), intent(in) : name ! component name

OUTPUT PARAMETERS:

! the local communicator of that component is written in comm.

integer, intent(out) : : comm ! communicator for the component

LOCAL VARIABLES:

integer : : id, $i$

5.1.6 PE_in_num_comps - return the number of components a processor in

This function returns the number of components a processor is in.

REVISION HISTORY:

2001-Dec-13 -- first prototype

INTERFACE:

integer function PE_in_num_comps ()

USES:

implicit none

SEE ALSO:

$\mathrm{PE}$ _in_component

LOCAL VARIABLES:

integer : : id, $i$ 


\subsubsection{MPH_global_id - find global processor id}

This function returns global processor id given the component name and local processor id in that component.

REVISION HISTORY:

2001-Nov-15 -- add PROTEX convention

2001-May-20 -- first prototype

INTERFACE:

integer function $\mathrm{MPH}_{-}$global_id (cname, lid)

USES:

implicit none

SEE ALSO:

MPH_find_name

INPUT PARAMETERS:

character(len=*), intent(in) :: cname ! component name

integer, intent(in) : : lid

! local processor id in the component

OUTPUT PARAMETERS:

! This function returns global_proc_id given the component

! name and local_proc_id in that component.

LOCAL VARIABLES:

integer : : temp

\subsubsection{MPH_comm_join - join two components}

This routine creates a joined MPI communicators for any two components. The order of these two components appeared in the subroutine parameter argumment has an effect on the local process id within the joined communicator.

\section{REVISION HISTORY:}


2001-Nov-15 -- add PROTEX convention

2001-May-20 -- first prototype

INTERFACE:

subroutine $\mathrm{MPH}_{-}$comm_join (name1, name2, comm_joined)

USES:

implicit none

SEE ALSO:

MPH_find_name, PE_in_component

INPUT PARAMETERS:

character(len=*), intent(in) : name1, name2 ! two component names

OUTPUT PARAMETERS:

integer, intent(out) : : comm_joined

! joined communicator for two components

LOCAL VARIABLES:

integer : : id1, id2

integer : : color, key

logical : : con1, con2

integer : : comm1, comm2

\subsubsection{MPH_redirect_output - redirect output from each component}

This routine redirects output to a $\log$ file defined by an environment variable. System functions ("getenv" for IBM and "pxfgetenv" for SGI and T3E) are used to retrieve the environmet variable.

REMARKS: 
In order to redirect component output to a separate file,

a user will setup something like the following in the run script:

setenv ice_out_env ice.log

setenv land_out_env land.log

setenv cpl_out_env cpl.log

REVISION HISTORY:

2001-Nov-15 -- add PROTEX convention

2001-May-20 -- first prototype

INTERFACE:

subroutine MPH_redirect_output (name)

USES:

implicit none

INPUT PARAMETERS:

character(len=*), intent(in) :: name ! part of the log file name

\section{LOCAL VARIABLES:}

integer : : lenname, lenval, rcode

character(len=32) : : output_name_env

character(len=64) : : output_name, temp_value

\subsubsection{MPH_read_list - read and process info from "processors_map.in"}

This routine reads and processes info from "processors_map.in". Please see a sample input

file in Introduction.

REVISION HISTORY:

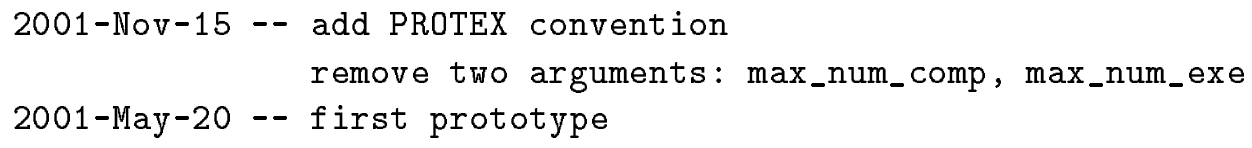

INTERFACE: 


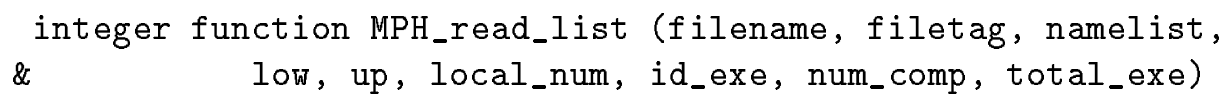

USES:

implicit none

SEE ALSO:

MPH_find_name, MPH_init

INPUT PARAMETERS:

character(len=*), intent(in):: filename ! the input file name

character(len=*), intent(in) : filetag ! "PROCESSORS_MAP"

OUTPUT PARAMETERS:

character (len=32), intent(out) : : namelist (max_num_comps)

! component names

integer, intent(out) : : low(max_num_comps)

! lower processor limit of each component

! in each executable world

integer, intent(out) : : up (max_num_comps)

! upper processor limit of each component

! in each executable world

integer, intent(out) : : local_num(max_num_comps)

! total number of processors for each component

integer, intent(out) : : id_exe(max_num_comps) ! executable ids

integer, intent(out) : : num_comp(max_num_comps)

! number of components in each executable

integer, intent(out) : : total_exe ! total number of executables

\section{LOCAL VARIABLES:}

integer : : $i, k$, multi_num, id

character (len=32) : : firstline, temp

integer :: itemp1, itemp2 


\subsubsection{MPH_find_name - find name in a namelist}

This routine finds if a certain name exists in an array of namelist and returns the rank if it does or -1 if it does not.

REVISION HISTORY:

2001-Nov-15 -- add PROTEX convention

2001-May-20 -- first prototype

INTERFACE:

integer function MPH_find_name (name, namelist, num)

USES:

implicit none

INPUT PARAMETERS:

character(len=*), intent(in):: name ! name to be found integer :: num ! length of namelist array

character (len=32) namelist(num) ! name list array

OUTPUT PARAMETERS:

! the rank of a name in an array of namelist or -1 if not exist

LOCAL VARIABLES:

integer : : i

\subsubsection{MPH_help - display help info}

This routine displays some help info for the MPH setup interface and some inquiry functions.

REVISION HISTORY:

2001-Nov-15 -- add PROTEX convention

2001-May-20 -- first prototype

INTERFACE: 
subroutine MPH_help (arg)

USES:

implicit none

INPUT PARAMETERS:

character(len=*), intent(in) : : arg ! either 'on' or 'off'

\subsubsection{MPH_debug - define debug level}

This routine defines the debug level. The higher the level is, the more debug information the code will display.

REVISION HISTORY:

2001-Nov-15 -- add PROTEX convention

2001-May-20 -- first prototype

INTERFACE:

subroutine $\mathrm{MPH}_{-}$debug (level)

USES:

implicit none

INPUT PARAMETERS:

integer, intent(in) :: level ! 0 (default), 1 or 2

\subsubsection{MPH_timer - collect timing info in different channels.}

This function collects timing info in different channels.

Usage:

channel 0 is the default channel, using init_time.

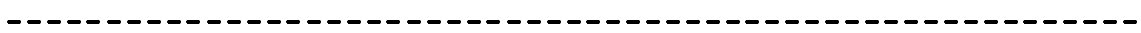

timer calls to walk-clock dclock(), and do the following: 


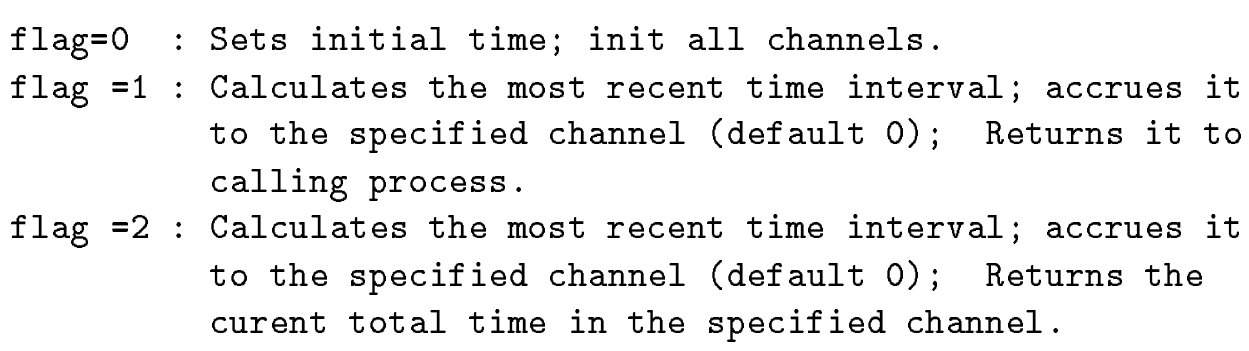

REVISION HISTORY:

2001-Nov-19 -- add PROTEX convention

2001-May-20 - - first prototype

INTERFACE:

real (kind=8) function MPH_timer (flag, channel)

USES:

implicit none

INPUT PARAMETERS:

integer : : flag, channel

OUTPUT PARAMETERS:

! real (kind=8) $\mathrm{MPH}_{-}$timer (flag, channel)

LOCAL VARIABLES:

real (kind=8) : : new_time, delta_time, MPI_Wtime

\subsubsection{MPH_total_components - find number of total components}

This function returns the number of total components.

REVISION HISTORY: 
2001-Nov-15 -- add PROTEX convention

2001-May-20 -- first prototype

INTERFACE:

integer function MPH_total_components ()

USES:

implicit none

OUTPUT PARAMETERS:

! total_components

\subsubsection{MPH_comp_name - find component name given component id}

This function returns component name given component id.

REVISION HISTORY:

2001-Dec-13 -- use optional argument

2001-Nov-15 -- add PROTEX convention

2001-May-20 -- first prototype

INTERFACE:

character (len=32) function MPH_comp_name (cid)

USES:

implicit none

SEE ALSO:

MPH_find_name, MPH_comp_id, MPH_comm

INPUT PARAMETERS:

integer, intent(in), optional : : cid ! component id 
OUTPUT PARAMETERS:

! component_names (cid)

LOCAL VARIABLES:

integer : : id, comm

\subsubsection{MPH_comp_id - find component id given component name}

This routine returns component id given component name.

\section{REVISION HISTORY:}

2001-Dec-13 -- use optional argument

2001-Nov-15 -- add PROTEX convention

2001-May-20 -- first prototype

INTERFACE:

integer function MPH_comp_id (cname)

USES:

implicit none

SEE ALSO:

MPH_find_name, MPH_comp_name, MPH_comm

INPUT PARAMETERS:

character(len=*), intent(in), optional :: cname ! component name

OUTPUT PARAMETERS:

! MPH_comp_id

LOCAL VARIABLES:

integer : : id, comm 


\subsubsection{MPH_local_world - find local communicator given component name}

This routine returns local communicator given component name.

REVISION HISTORY:

2001-Dec-13 -- first prototype

INTERFACE:

integer function MPH_local_world (cname)

USES:

implicit none

SEE ALSO:

MPH_find_name, MPH_comp_id

INPUT PARAMETERS:

character(len=*), intent(in), optional :: cname ! component name

OUTPUT PARAMETERS:

! MPH_local_world

LOCAL VARIABLES:

integer : : id, comm

\subsubsection{MPH_exe_id - find executable id given component name}

This function returns the executable id given component name.

REVISION HISTORY:

2001-Dec-13 -- use optional argument

2001-Nov-15 -- add PROTEX convention

2001-May-20 -- first prototype 
INTERFACE:

integer function MPH_exe_id (cname)

USES:

implicit none

SEE ALSO:

MPH_find_name

INPUT PARAMETERS:

character(len=*), intent(in), optional : cname ! component name

OUTPUT PARAMETERS:

! exe_ids (id)

!LOCAL PARAMETERS:

integer : : id, comm

\subsubsection{MPH_total_num_exe - find total number of executables}

This fuction returns the total number of executables.

REVISION HISTORY:

2001-Nov-15 -- add PROTEX convention

2001-May-20 -- first prototype

INTERFACE:

integer function MPH_total_num_exe ()

USES:

implicit none

OUTPUT PARAMETERS:

! total_num_exe 


\subsubsection{MPH_num_comps - find number of components in an executable}

This fuction returns number of components in an executable given the executable id.

REVISION HISTORY:

2001-Dec-13 -- use optional argument

2001-Nov-15 -- add PROTEX convention

2001-May-20 -- first prototype

INTERFACE:

integer function MPH_num_comps (eid)

USES:

implicit none

INPUT PARAMETERS:

integer, intent(in), optional : eid ! executable id

OUTPUT PARAMETERS:

! num_comps (eid)

\subsubsection{MPH_local_proc_id - find local processor id in a component}

This function returns the local processor id given the component id.

REVISION HISTORY:

2001-Dec-13 -- use optional argument

2001-Nov-15 -- add PROTEX convention

2001-May-20 -- first prototype

INTERFACE:

integer function MPH_local_proc_id (cid)

USES:

implicit none 
SEE ALSO:

MPH_global_proc_id

INPUT PARAMETERS:

integer, intent(in), optional : cid ! component id

OUTPUT PARAMETERS:

! local_proc_id (cid)

LOCAL VARIABLES:

integer : : id, comm

\subsubsection{MPH_local_totProcs - find total number of processors}

in a component.

This function returns the total number of processors in a component given the component id.

REVISION HISTORY:

2001-Dec-13 -- use optional argument

2001-Dec-13 -- use optional argument

2001-Nov-27 -- first prototype

INTERFACE:

integer function MPH_local_totProcs (cid)

USES:

implicit none

SEE ALSO:

MPH_global_totProcs

INPUT PARAMETERS: 
integer, intent(in), optional : cid ! component id

OUTPUT PARAMETERS:

! local_totProcs (cid)

LOCAL VARIABLES:

integer : : id, comm

\subsubsection{MPH_global_proc_id - find global processor id}

This function returns the global processor id.

REVISION HISTORY:

2001-Nov-15 -- add PROTEX convention

2001-May-20 -- first prototype

INTERFACE:

integer function MPH_global_proc_id ()

USES:

implicit none

SEE ALSO:

MPH_local_proc_id

OUTPUT PARAMETERS:

! global_proc_id 


\subsubsection{MPH_global_proc_id - find total number of processors}

This function returns the total number of processors in MPH world.

REVISION HISTORY:

2001-Nov-15 -- add PROTEX convention

2001-May-20 -- first prototype

INTERFACE:

integer function MPH_global_totProcs ()

USES:

implicit none

OUTPUT PARAMETERS:

! global_totProcs

\subsubsection{MPH_local_world - find local communicator of an executable}

This function returns the local MPI communicator of an executable given the executable id.

REVISION HISTORY:

2001-Dec-13 -- change function name from MPH_local_world to MPH_exe_world, use optional argument

2001-Nov-15 -- add PROTEX convention

2001-May-20 - - first prototype

INTERFACE:

integer function MPH_exe_world (eid)

USES:

implicit none

INPUT PARAMETERS: 
integer, intent(in), optional : : eid ! executable id

OUTPUT PARAMETERS:

! exe_world (eid)

\subsubsection{MPH_exe_low_proc_limit - find lower processor limit of a component}

This function returns the relative lower processor limit of a component in the executable world.

REVISION HISTORY:

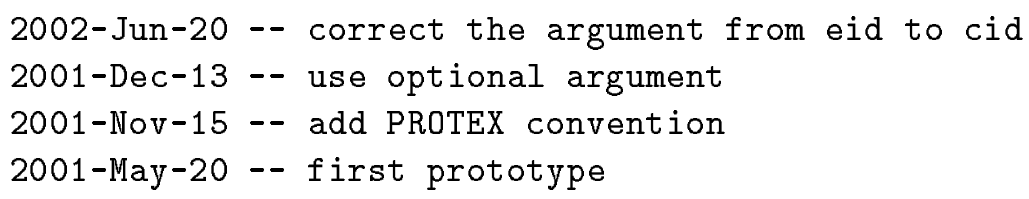

INTERFACE:

integer function $\mathrm{MPH}_{-}$exe_low_proc_limit (cid)

USES:

implicit none

SEE ALSO:

MPH_exe_up_proc_limit

INPUT PARAMETERS:

integer, intent(in), optional : cid ! component id

OUTPUT PARAMETERS:

! exe_low_proc_limit (cid) 


\subsubsection{MPH_exe_up_proc_limit - find upper processor limit of a component}

This function returns the relative upper processor limit of a component in the executable world.

\section{REVISION HISTORY:}

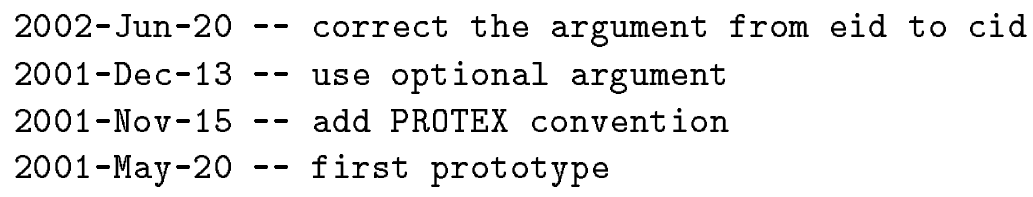

INTERFACE:

integer function MPH_exe_up_proc_limit (cid)

USES:

implicit none

SEE ALSO:

MPH_exe_low_proc_limit

INPUT PARAMETERS:

integer, intent(in), optional : : cid ! component id

OUTPUT PARAMETERS:

! exe_up_proc_limit (cid) 